\title{
Neuroprotective Effects of Lycium Barbarum in Ischemic Stroke: Current Perspectives
}

\section{Sarode LP, Mardhekar VY and Ugale RR*}

Department of Pharmaceutical Sciences, Rashtrasant Tukadoji Maharaj Nagpur University, Nagpur, Maharashtra, India

*Corresponding author: Dr. Ugale RR, Department of Pharmaceutical Sciences, Rashtrasant Tukadoji Maharaj Nagpur University, Nagpur, Maharashtra, India, Email: ugale.rajesh@gmail. com

\section{Abstract}

Lycium barbarum is well known traditional medicine used for centuries as nutritive agent. It has been extensively studied and proved as immunomodulatory, antiaging, antihyperglycemic, antitumour, cytoprotective and neuroprotective in nature. Lycium barbarum has strong free radical scavenging and antioxidant property. It bears antiinflammatory and antimicrobial potential. Recently, Lycium barbarum has been reported to inhibit glutamate excitotoxicity by regulating NR2B and NR2A signaling pathways. In addition, it regulates PI3K/Akt/mTOR signaling pathway which inhibits apoptosis and also showed free radical scavenging activity which may prevent further neuronal cell death. However, exact mechanism of Lycium barbarum which improves ischemic neuronal cell death is still under investigation. Ischemic stroke is a leading cause of death and morbidity worldwide due to lack of clinically effective therapy. Ischemic stroke and ischemic reperfusion injury has complex pathogenesis which includes mainly glutamate excitotoxicity, apoptosis and oxidative stress. However, neuroprotection in ischemic stroke appears to be emerging strategy for the development of therapeutic agents with fewer side effects. This review presents pharmacological mechanisms of Lycium barbarum elucidated in latest research reports and its action in ischemic stroke. Lycium barbarum may provide potential alternative or an adjunct therapy to prevent ischemic stroke complications.

Keywords: Lycium Barbarum; Ischemic Stroke; Neuroprotection; Traditional Medicine; Antioxidant

\section{Introduction}

Ischemic stroke leads to permanent damage of brain resulting in long-term disability and death worldwide [1]. Intravenous recombinant tissue plasminogen activator (rtPA) is the only effective American Food and Drug Administration (FDA)-approved pharmacological treatment for ischemic stroke, the clinical effectiveness of which is extremely limited, owing to the short therapeutic window and an increased risk of subarachnoid haemorrhage [2]. Neuronal cell death in stroke has been recognized as a result of focal ischemic damage to secondary brain injury and interlinking pathways. Several events like glutamate excitotoxicity, oxidative stress, and inflammation predominate at the core of ischemic brain damage. Reports have shown that peripheral region of core also known as ischemic penumbra may undergo apoptosis after several hours or days and thus provide sufficient time to recover by preventing further apoptotic pathways within time frame after ischemic insult [2]. Thus, neuroprotection offers an emerging strategy towards the development of potential therapeutic agents for ischemic stroke. This strategy emphasizes on preventing progression of neuronal cell death, through actions on molecular Pathways involved in ischemic pathogenesis rather than by improving blood flow [3]. In these context natural compounds derived from traditional medicines offers several advantages, due to abundant available resources, multi-targeted mechanisms of activity, few side-effects, and no drug resistance [4]. In this 
review, Lycium barbarum, also known as wolfberry, a wellknown traditional Chinese medicine used for centuries in many Asian countries for health improvement and as a food supplement has been explored for its role in neuroprotection.

\section{Lycium barbarum: Source and Availability}

The genre Lycium includes more than 90 species among which galophytes occur. Among available species, $L$. afrum, L. andersonii, L. arabicum, L. australe, L. barbarum, L. berlandieri, L. carolinianum, L. chinense, L. europaeum, L. fremontii, L. pallidum, L. ruthenicum, L. schweinfurthii, $L$. Torreyi species grows mainly in Asia. The wolfberry (Lycium barbarum L.) belongs to the Solanaceae distributed in arid and subarid regions in temperate to subtropical zones in South America, North America, South Africa, Eurasia and Australia. It is also known as Fruktus Iycii, Gougizi, and Goji berry in China and as wolfberry in Europe and North America. China is currently the greatest supplier of wolfberry in the world. The wolfberry has been widely used as a traditional Chinese herb and functional food in China and other Asian countries including Vietnam, Korea, and Japan for more than 2500 years [5].

\section{Active Phytochemicals of Lycium Barbarum}

Lycium barbarum has been reported as an exotic super food due to its high amount of polysaccharide, vitamin, and carotenoid content. Approximately $5 \%-8 \%$ of the dry weight of Lycium barbarum consists of Lycium barbarum polysaccharides or glycoconjugates (LBP). LBP also includes galacturonic acid and 18 amino acids along with high amount of xylose and glucose, and low amounts of arabinose, rhamnose, mannose, and galactose. Furthermore, Lycium barbarum fruit also includes scopoletin (6-methoxy-7hydroxycoumarin, also named chrysatropic acid, ecopoletin, gelseminic acid, and scopoletol), vitamin $C$ analog 2-0- $\beta-$ Dglucopyranosyl-L-ascorbic acid, carotenoids (zeaxanthin and $\beta$-carotene), betaine, cerebroside, $\beta$-sitosterol, flavonoids, amino acids. It is also the source of 21 trace minerals including zinc, iron, copper, calcium, selenium, and phosphor, and also thiamin (B1), riboflavin (B2), pyridoxine (B6), vitamin E, and vitamin C [6,7].

As the wolfberry contains both water soluble (e.g., LBP, vitamin C precursor) and fat-soluble (e.g., zeaxanthin) phytochemicals, the bioavailability and nutritional properties are likely to be affected by the method of extraction. For wolfberry, the use of hot water allows the extraction of hydrophilic compounds, but most of the lipophilic components such as zeaxanthin dipalmitates, lipophilic vitamins, and other lipids are lost. Consuming purified extracts of phytonutrients is less beneficial than consuming the wolfberry in which complete phytonutrients are found. Therefore, identifying the most suitable extraction process, analyzing the chemical stability and bioavailability of the extracts is the need of the hour. There are practically no reports of adverse effects of wolfberry. Only a few cases of allergic reactions including urticaria-like or papular rashes have been documented. At very high dose adverse effects such as vertigo, palpitations, nausea, vomiting, and premature contractions have been reported otherwise this considered as completely safe $[6,7]$.

\section{Role in Neuroprotection}

Lycium barbarum and LBP are reported to exhibit neuroprotective effects against ageing-related neurodegenerative diseases. Lycium barbarum and an arabinogalactan-protein (LBP-III) exhibited cytoprotective effects by reducing the phosphorylation of doublestranded RNA-dependent protein kinase (PKR) triggered by beta-amyloid peptide, and lowering the dithiothreitol (DTT)-induced LDH release and caspase-3 activity, but not caspase-8 and -9 [8]. Similarly, pre-treatment of aqueous Lycium barbarum extract also reduced the phosphorylation of c-Jun N-terminal kinase (JNK)-1 (Thr183/ Tyr185) and its substrates c-Jun-I (Ser 73) and c-Jun-II (Ser 63), which are rapidly activated by beta-amyloid [9]. An alkaline extract of Lycium barbarum protected neurons from beta-amyloid peptide neurotoxicity. Moreover, some of these fractions markedly enhanced the phosphorylation of Akt [10]. This neuroprotective effect may come from both antioxidative and cytoprotective mechanisms, and by inhibiting proapoptotic signaling pathways. Thus Lycium barbarum may be a potential neuroprotective agent against Alzheimer's disease [11].

Elevated plasma homocysteine levels and glutamate excitotoxicity are suggested to increase the risk of neurodegenerative diseases by inducing apoptosis, DNA fragmentation, and hyperphosphorylation. LBP also exerted neuroprotective effects against homocysteine and glutamate excitotoxicity [12]. The Purified Lycium barbarum polysaccharide (LBPS02) successfully demonstrated neuroprotective effect against glutamate (L-Glu)-induced differentiated PC12 (DPC12) cell apoptosis. It was found that LBPS02 normalized the levels of anti-apoptotic proteins and regulated the phosphorylation of extracellular signalregulated kinases (ERKs) and protein kinase B (Akt) in L-Glu-explored DPC12 cells. It also significantly increased the levels of innate antioxidant enzymes GSH- Px, SOD and CAT in glutamate-induced PC12 cells [13].

Recently, it has been reported that LBP has neurotoxicity reversing potential. LBP concentration-dependently reversed the $\mathrm{H}_{2} \mathrm{O}_{2}$-induced increase in reactive oxygen species (ROS) levels, decrease in cell viability, increase in caspase-3 and -9 
activity and decrease in mitochondrial membrane potential, indicating the amelioration of mitochondrial apoptosis. Furthermore, silencing of Nrf2 and inhibition of HO-by zinc protoporphyrin IX (ZnPP) reversed the protective effects of LBP against $\mathrm{H}_{2} \mathrm{O}_{2}-$, $\mathrm{CoCl}_{2}$ - induced neurotoxicity in PC12 cells. This confirms that LBP inhibited the H2O2-induced decrease in nuclear factor erythroid 2-related factor $2(\mathrm{Nrf}) 2$ and heme oxygenase (HO)-1 expression and binding of Nrf2 to the promoters of HO-1 [14,15].

Lycium barbarum and LBP have been reported in vivo to have neuroprotective effects against various toxins and conditions enhanced the learning and memory capability of manganese-poisoned mice by promoting neurogenesis in the hippocampus [16]. Lycium barbarum has been reported to prevent brain oxidative mitochondrial damage in a prenatal stress model with rats and cognitive dysfunction associated with prenatal stress.

\section{Lycium Barbarum and Ischemic Stroke}

Ischemic stroke results from sudden interruption in cerebral blood flow, caused by occlusion of a cerebral artery by an embolus or local thrombosis. Penumbra often progresses to infarction owing to the effects of ongoing excitotoxicity, apoptosis and post-ischemic inflammation. Maintenance of survival of neurons within this dynamic area of tissue is critical to reduce permanent damage $[17,18]$. The neuroprotective effect of the kinase cascade including phosphoinositide 3 kinase (PI3K), protein kinase B (Akt), and the mammalian target of rapamycin (mTOR) (PI3K/Akt/ mTOR) signaling pathway has been widely studied in cerebral ischemia research. This is the central cascade involved in cell transcription, translation, migration, metabolism, proliferation, and survival. The pathway is highly related to apoptosis and autophagy. Several studies have demonstrated that the PI3K/Akt/mTOR signaling pathway also inhibits apoptosis, promotes the cell cycle, and inhibits autophagy when it is activated. It has been demonstrated that Lycium barbarum exert neuroprotective effects by regulating the PI3K/Akt/mTOR signaling pathway. In addition, LBP caused the down-regulation of cleaved Caspase-3/Caspase-3, LC3II/ LC3I and Beclin 1, as well as up-regulation of Bcl-2/Bax and p62 [19].

Moreover, it has been reported that effect of LBP against ischemic injury can be achieved by regulating NR2B and NR2A signaling pathways. LBP substantially reduced CA1 neuronal death after transient global ischemia and ameliorated memory deficit in ischemic rats. in vitro, LBP increased the viability of primary cultured cortical neurons when exposed to oxygen-glucose deprivation (OGD) for 4h. Importantly, LBP antagonized increase in expression of major proteins in the NR2B signal pathway including NR2B,
nNOS, Bcl-2-associated death promoter (BAD), cytochrome $\mathrm{C}$ (cytC) and cleaved caspase- 3, and also reduced ROS level, calcium influx and mitochondrial permeability after $4 \mathrm{~h}$ OGD. In addition, LBP prevented the down regulation in the expression of NR2A, pAkt and pCREB, which are important cell survival pathway components. Furthermore, LBP attenuated the effects of NR2B co-agonist and NR2A inhibitor on cell mortality under OGD conditions. Taken together, LBP proven to be neuroprotective against ischemic injury by its dual roles in activation of NR2A and inhibition of NR2B signaling pathways. Thus LBP exhibit potential for the treatment of ischemic stroke [20].

LBP markedly improved neurologic deficits and decreased infarct size and water contents at $24 \mathrm{~h}$ after reperfusion in mice. Pathological section of brain tissues also proved its protective effects on neurocytes. LBP markedly down-regulated the protein level of NF- $\kappa B$, p65. LBP decreased the levels of TNF- $\alpha$, IL- 6 and IL- $1 \beta$ in the serum 24 $\mathrm{h}$ after reperfusion. Thus LBP showed protective effects on cerebral ischemia-reperfusion injury [21].

LBP treatment prevented the HG-induced alterations in Drp-1 and Opa1 expression. Author showed that LBP reduced increase in phospho-Drp1 caused by HG. These results suggest that pre-treatment with LBP ameliorates the hyperglycemia-enhanced ischemic brain damage through maintaining mitochondrial dynamic balance [22].

In addition, LBP pretreatment significantly enhanced regional cortical blood flow and the total power of the spontaneous EEG, improved memory and motor coordination impairments, and inhibited over-activation of microglia and astrocytes after MCAO. Further research demonstrated LBP suppressed MCAO-induced activations of P65 NF- $\mathrm{KB}$ and P38 MAPK, and prevented up-regulation of proinflammatory mediators in hippocampus. Lycium barbarum has also been found to inhibit erythrocyte hemolysis, prolong low-density lipoprotein oxidation and inhibit vascular smooth muscle cell migration through the MAPK pathway, which are important pathologic events during the initiation of atherosclerosis and stroke [23].

Moreover, LBP can exert functional recovery of memory and motor coordination deficits and neuroprotective effect against cerebral ischemic injury in mice. LBP (10-40 mg/l) significantly attenuated neuronal damage and inhibited LDH release in a dose-dependent manner. Furthermore, LBP enhanced activities of SOD and GSH-PX but it decreased their MDA content, inhibited calcium elevation and decrease of MMP in ischemia-reperfusion treated hippocampal neurons. LBP prevent the cerebral reperfusion-induced injury in the brain by reducing lipid peroxides, scavenging free radicals, and improving the energy metabolism $[24,25]$. 


\begin{tabular}{|c|c|c|}
\hline Properties & Relationship & Reference \\
\hline \multirow[b]{2}{*}{ Antioxidant } & $\begin{array}{l}\text { LBP treatment significantly increased Nrf2 nuclear accumulation and } \\
\text { HO-1 expression in the retina after I/R injury. }\end{array}$ & \multirow[t]{2}{*}{ [15] } \\
\hline & $\begin{array}{l}\text { LBP enhanced activities of SOD and GSH-PX but it decreased their } \\
\text { MDA content, inhibited }[\mathrm{Ca}(2+)](\mathrm{i}) \text { elevation and decrease of MMP in } \\
\text { ischemia-reperfusion treated hippocampal neurons. }\end{array}$ & \\
\hline Action on glutamate excitotoxicity & $\begin{array}{l}\text { LBP antagonized increase in expression of major proteins in the NR2B } \\
\text { signal pathway including NR2B, nNOS, Bcl-2-associated death pro- } \\
\text { moter (BAD), cytochrome C (cytC) and cleaved caspase-3, and also } \\
\text { reduced ROS level, calcium influx and mitochondrial permeability. } \\
\text { In addition, LBP prevented the downregulation in the expression of } \\
\text { NR2A, pAkt and pCREB, which are important cell survival pathway } \\
\text { components }\end{array}$ & {$[20]$} \\
\hline Antiinflammatory & $\begin{array}{l}\text { LBP markedly down-regulated the protein level of NF- } \kappa \text { B, p } 65 \text { along } \\
\text { with decreased the levels of TNF- } \alpha \text {, IL- } 6 \text { and IL-1 } \beta\end{array}$ & {$[21]$} \\
\hline Antihyperglycemic & $\begin{array}{l}\text { Pre-treatment with LBP ameliorates the hyperglycemia-enhanced } \\
\text { ischemic brain damage through maintaining mitochondrial dynamic } \\
\text { balance that is decrease in phospho-Drp1 and increase in Opa1 }\end{array}$ & {$[22]$} \\
\hline Action on Learning and memory & $\begin{array}{l}\text { LBP pretreatment significantly enhanced regional cortical blood flow } \\
\text { and the total power of the spontaneous EEG, improved memory and } \\
\text { motor coordination impairments, and inhibited over-activation of } \\
\text { microglia and astrocytes after ischemia }\end{array}$ & \multirow[b]{2}{*}{ [19] } \\
\hline \multirow[t]{2}{*}{ Antiapoptotic } & $\begin{array}{l}\text { LBP protects against cerebral ischemia/reperfusion-induced in pri- } \\
\text { mary hippocampal neurons through the down-regulation of cleaved } \\
\text { Caspase-3/Caspase-3, LC3II/LC3I and Beclin 1, as well as up-regula- } \\
\text { tion of Bcl-2/Bax and p62 as well as increase in p-Akt and p-mTOR } \\
\text { levels after OGD/R. }\end{array}$ & \\
\hline & $\begin{array}{l}\text { LBP suppressed overexpression of Bax, CytC, Caspase- } 3,-9 \text { and } \\
\text { cleaved PARP-1, and inhibited the reduction of Bcl-2 expression. }\end{array}$ & {$[25]$} \\
\hline
\end{tabular}

Table 1: Summary of recent leading neuroprotective studies on Lycium barbarum.

\section{Conclusion}

This review concludes that Lycium barbarum exhibits the proven neuroprotective properties in ischemic stroke. Although the exact mechanism is yet unknown, several mechanisms are well established such as the attenuation of cellular oxidative stress, inflammation, apoptosis and the improvement of neuronal function. Besides, the key functions of the MAPK pathway, such as ERK and JNK, PI3K/ Akt/mTOR pathways are repetitively presented in those studies. Literature clearly shows that the neuroprotective role of Lycium barbarum is primarily due to inhibition of glutamate excitotoxicity, through regulating NR2A/NR2B signaling pathway. However, future studies are warranted for the neuroprotective effects of Lycium barbarum in ischemic stroke before its clinical use. Further investigations on pharmacokinetics including studies on the bioavailability and pharmacodynamics of Lycium barbarum and its derivatives in neurological disorders, improvement in bioavailability, finding the optimum effective dose for the neuroprotective effect are needed. Taken together Lycium barbarum may emerge as a novel therapeutic agent for ischemic stroke therapy.

\section{Conflicts of Interest}

The author(s) confirm that this article content has no conflicts of interest.

\section{References}

1. Della Morte D, Guadagni F, Palmirotta R, Testa G, Caso $V$, et al. (2012) Genetics of ischemic stroke, strokerelated risk factors, stroke precursors and treatments. Pharmacogenomics 13(3): 595-613.

2. Del Zoppo G, Saver J, Jauch E, Adams H (2009) Expansion of the time window for treatment of acute ischemic stroke with intravenous tissue plasminogen activator: a 
science advisory from the American Heart Association/ American Stroke Association. Stroke 40(8): 2945-2948.

3. Maria A, Joan M (2015) Advanced neuroprotection for brain ischemia: an alternative approach to minimize stroke damage. Expert Opin Investig Drugs 24(9): 11371142.

4. Xiong X, Liu L, Yang Q (2018) Refocusing neuroprotection in cerebral reperfusion era: New challenges and strategies. Front Neurol 9: 249.

5. Serebryanaya F, Sekinayeva M, Denisenko O (2018) Comparative micromorphological investigations of red godji berries (Lycium barbarum L.) and black godji berries (lycium ruthenicummurr.). Pharmacog J 10(5): 911-915.

6. Harunobu A, Norman R (2011) Review of botanical characteristics, phytochemistry, clinical relevance in efficacy and safety of Lycium barbarum fruit (Goji). Food Res Int 44(7): 1702-1717.

7. Potterat O (2010) Goji (Lycium barbarum and L. chinense): Phytochemistry, pharmacology and safety in the perspective of traditional uses and recent popularity. Planta Med 76(1): 7-19.

8. Ho Y, Yu M, Yang X, So K, Yuen W, et al. (2010) Neuroprotective effects of polysaccharides from wolfberry, the fruits of Lyciumbarbarum, against homocysteine-induced toxicity in rat cortical neurons. J Alzheimers Dis 19(3): 813-827.

9. Yu M, Leung S, Lai S, Che C, Zee S, et al. (2005) Neuroprotective effects of anti-aging oriental medicine Lyciumbarbarumagainstbeta-amyloid peptide neurotoxicity. Exp Gerontol 40(8-9): 716-727.

10. Ho Y, Yu M, Lai C, So, K, Yuen W, etal. (2007) Characterizing the neuroprotective effects of alkaline extract of Lycium barbarum on beta-amyloid peptide neurotoxicity. Brain Res 1158: 123-134.

11. Yu M, Ho Y, So K, Yuen W, Chang R (2006) Cytoprotective effects of Lycium barbarum against reducing stress on endoplasmic reticulum. Int J Mol Med 17(6): 1157-1161.

12. Ho $Y, Y u$ M, Yik S, So K, Yuen $W$, et al. (2009) Polysaccharides from wolfberry antagonizes glutamate excitotoxicity in rat cortical neurons. Cell Mol Neurobiol 29(8): 1233-1244.

13. Kou L, Du M, Zhang C, Dai Z, Li X, et al. (2017) Polysaccharide purified from Lycium barbarum protects differentiated PC12 cells against L-Glu-induced toxicity via the mitochondria-associated pathway. Mol Med Rep
16(4): 5533-5540.

14. Cao S, Du J, Hei Q (2017) Lycium barbarum polysaccharide protects against neurotoxicity via the Nrf2-HO-1 pathway. Exp Ther Med 14(5): 4919-4927.

15. He M, Pan H, Chang R, So $K$, Brecha N, et al. (2014) Activation of the Nrf2/HO-1 antioxidant pathway contributes to the protective effects of Lycium barbarum polysaccharides in the rodent retina after ischemiareperfusion-induced damage. Plosone 9(1): e84800.

16. Wen J, Yang B, Ren D (2010) Effect of Lycium barbarum polysaccharides on neurogenesis and learning \& memory in manganese poisoning mice. Chin J Integr Med 30(3): 295-298.

17. Hai-Yang G, Ping S, Li J, Chang-Hong X, Fu T (2004) Therapeutic effects of Lycium barbarum polysaccharide (LBP) on mitomycin C (MMC)-induced myelosuppressive mice. J Exp Ther Oncol 4(3): 181-187.

18. Slevin M, Krupinski J, Kumar P, Gaffney J, Kumar $S$ (2005) Gene activation and protein expression followingischaemic stroke: strategies towards neuroprotection. J Cell Mol Med 9(1): 85-102.

19. Yu Y, Wu X, Pu J, Luo P, Ma W, et al. (2018) Lycium barbarum polysaccharide protects against oxygen glucose deprivation/reoxygenation-induced apoptosis and autophagic cell death via the PI3K/Akt/mTOR signaling pathway in primary cultured hippocampal neurons. Biochem Biophys Res Commun 495(1): 11871194.

20. Shi Z, Zhu L, Li T, Tang X, Xiang Y, et al. (2017) Neuroprotective mechanisms of Lycium barbarum polysaccharides against ischemic insults by regulating NR2B and NR2A containing NMDA receptor signaling pathways. Front Cell Neurosci 11: 288.

21. Ge J, Lu H, Song X, Li M, Chen D, et al. (2017) Protective effects of LBP on cerebral ischemia reperfusion injury in mice and mechanism of inhibiting NF- $\kappa B$, TNF- $\alpha$, IL- 6 and IL-1 $\beta$. Zhongguo Zhong Yao Za Zhi (article in Chinese) 42(2): 326-331.

22. Liu W, Jiang H, Rehman F, Zhang J, Chang Y, et al. (2017) Lycium barbarum polysaccharides decrease hyperglycemia-aggravated ischemic brain injury through maintaining mitochondrial fission and fusion balance. Int J Biol Sci 13(7): 901-910.

23. Zhao P, Zhou R, Zhu X, Liu G, Zhao Y, et al. (2017) Neuroprotective effects of Lycium barbarum polysaccharide on focal cerebral ischemic injury in mice. 
Neurochem Res 42(10): 2798-2813.

24. Rui C, Yuxiang L, Yinju H, Qingluan Z, Yang W, et al. (2012) Protective effects of Lycium barbarum polysaccharide on neonatal rat primary cultured hippocampal neurons injured by oxygen-glucose deprivation and reperfusion. J Mol Histol 43(5): 535-542.
25. Wang T, Li Y, Wang Y, Zhou R, Ma L, et al. (2014) Lycium barbarum Polysaccharide Prevents Focal Cerebral Ischemic Injury by Inhibiting Neuronal Apoptosis in Mice. PLoS ONE 9(3): e90780. 